\title{
Excavation mechanics of the elongated female rostrum of the acorn weevil Curculio glandium (Coleoptera; Curculionidae)
}

\author{
Yoko Matsumura ${ }^{1}$ (D) Mohsen Jafarpour ${ }^{1,2}$ - Michał Reut ${ }^{3} \cdot$ Bardiya Shams Moattar $^{2,4} \cdot$ Abolfazl Darvizeh $^{2} \cdot$ \\ Stanislav N. Gorb ${ }^{1}$ (D) Hamed Rajabi ${ }^{1}$ [D
}

Received: 31 October 2020 / Accepted: 5 February 2021 / Published online: 16 April 2021

(c) The Author(s) 2021

\begin{abstract}
Elongated rostra (snouts) are remarkable features of many female weevils. The female of Curculio glandium uses the snout to excavate channels in acorns to oviposit. Considering the slenderness of the rostrum, the excavation of channels in solid substrates without buckling is a challenging task from both engineering and biological points of view. Here we aimed to examine the roles of the material properties and morphology of the rostrum in its buckling resistance. We employed microscopy techniques, non-destructive material characterisation and finite element (FE) modelling to shed more light on the excavation mechanics of the rostrum. We found that sexual dimorphisms are present not only in the length but also in the material, particularly the elastic modulus, and morphological features, particularly the curvature and thickness of the cuticular layers. Our FE modelling showed that those factors play essential roles to maximise the buckling resistance and minimise the bending resistance of the female rostrum. Considering that during excavation, the rostrum needs to be straightened without buckling, the functionality of the rostrum is likely to be a compromise between the flexibility and stiffness.
\end{abstract}

Keywords Beetle $\cdot$ Biomechanics $\cdot$ Buckling prevention $\cdot$ Cuticle $\cdot$ Finite element $\cdot$ Bending

\section{Introduction}

The evolution of the elongated rostrum is a remarkable feature of herbivorous curculionoid beetles, called weevils. Except for the early split weevil families, i.e. Nemonychidae and Anthribidae, females process their host plants and prepare their oviposition sites using their rostrum before laying eggs [1, 2]. Anderson [1] posited oviposition in plant tissues with the aid of the rostrum as one of the key innovations

Hamed Rajabi

harajabi@hotmail.com; hrajabi@zoologie.uni-kiel.de

1 Department of Functional Morphology and Biomechanics, Zoological Institute, Kiel University, Am Botanischen Garten 1-9, 24118 Kiel, Germany

2 Faculty of Mechanical Engineering, University of Guilan, Rasht, Iran

3 Section of Applied Entomology, Department of Plant Protection, Institute of Horticulture Sciences, Warsaw University of Life Sciences, Warsaw, Poland

4 College of Engineering and Computing Sciences, Department of Energy Management, New York Institute of Technology, Vancouver V7Y 1K8, Canada of the weevil evolution. Weevils are indeed a megadiverse group and feed on a great variety of plant taxa and tissues [2]. The species richness is associated with host plant and feeding habit shifts [3], and especially the diversity of the weevil rostrum in shape and size along with the head morphology is conspicuous $[4,5]$.

The genus Curculio, to which our study species C. glandium belongs, shows remarkable variations in the length and curvature of rostra among species and between the sexes. Female weevils' rostra are often longer than that of males $[1,6]$. Females excavate channels in fruits using their elongated rostra, to lay eggs inside the fruits [1]. Hughes and Vogler [6] found a co-evolutionary pattern between the rostrum length and nuts size, suggesting that adaptations to host plants promoted the diversity of the rostrum length among species. Arms races between fruits and weevils are the primary driving force of the rostrum diversification, along with climatic conditions [7, 8].

The rostra of some Curculio species exhibit high aspect ratios and are seemingly fragile (see for example Fig. 1 in Toju and Sota [8], Fig. 49 in Davis [4] and Fig. 1 in Jansen et al. [9]). Excavation of channels in solid substrates using such slender tubes without buckling is a challenge also 
Fig. 1 Images of the rostrum and excavation behaviour of Curculio glandium. a A female (above) and male (bottom) heads. b Just after the female started excavation. c, d The rostrum is deeply inserted $\mathbf{c}$, and the female rotated her head almost $180^{\circ} \mathbf{d}$. e The female pulls her rostrum out

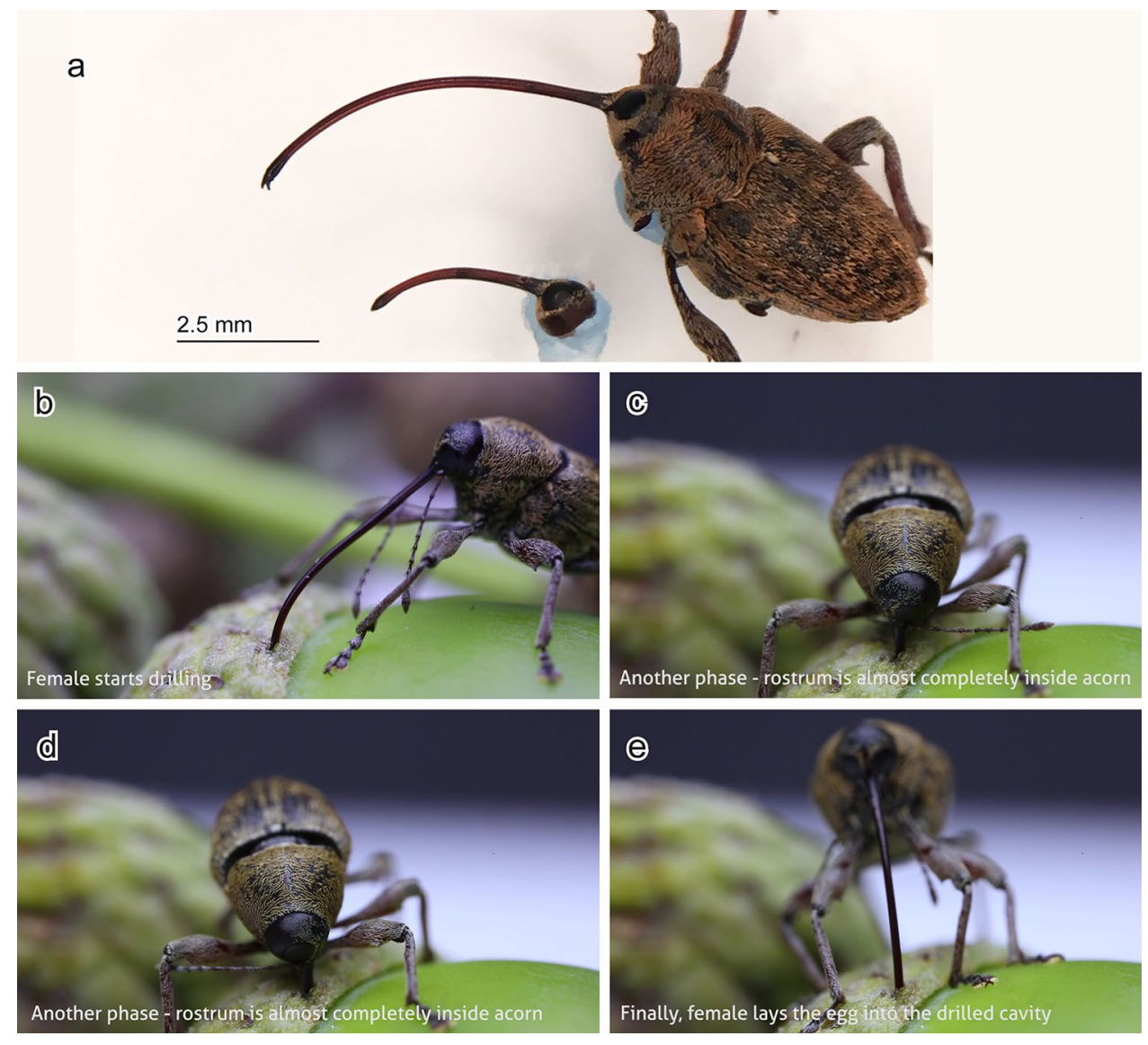

from an engineering point of view. Insects overcome this challenge with a variety of structural and material strategies (reviewed in $[10,11]$ ). Unlike other steerable probes in insects, such as ovipositors and mouthparts, which are composed of multiple sliding elements that support each other (summarised in Cerkvenik et al. [11]), movable mouthparts in weevils are situated at the tip of the rostrum and used to rasp or scrape substrates [4]. During excavation, female weevils push their rostra into acorns. Hence, the rostra are likely to withstand substantial compressive and bending loads. Singh et al. [12] and Jansen et al. [9, 13] examined the rostrum biomechanics in the North American Curculio weevils. Their studies showed that a cuticular macrofibre laminae arrangement and the presence of a large soft core-endocuticle_reduce the risk of buckling failure in the rostrum. However, taking into account that buckling prevention strategies are often complicated in nature $[10$, $11]$, the role of the material properties and shape of a loaded structure on the buckling resistance of the rostrum remains to be examined. Moreover, the link between the remarkable sexual dimorphism in the rostrum shapes and their biomechanics is entirely unexplored.

To fill this knowledge gap, in the present study, we explored the material and geometric aspects of the sexual dimorphism of the rostrum, and their effects on its mechanical performance. For this purpose, first, we analysed the geometry, microstructure and material distributions along rostra of the male and female using microscopy techniques and non-destructive material characterisation. Based on the results, we simulated the mechanical performance, i.e. bending and buckling resistance, of different sets of rostrum models using the finite element (FE) modelling. The FE results were used to understand how certain material and geometric features influence the biomechanics of female rostra under loading. We then discussed the excavation mechanics and potential mechanical adaptations of the rostra in an evolutionary context.

\section{Materials and methods}

\subsection{Samples}

The study weevil, Curculio glandium, was collected at the beginning of their reproductive seasons, in June 2016 and 2017 at the campus of Warsaw University of Life Sciences, Warsaw, Poland (52 $\left.10^{\prime} 1.76^{\prime \prime} \mathrm{N}, 21^{\circ} 2^{\prime} 48.31^{\prime \prime E}\right)$. Before experiments, the living weevils were kept in small plastic containers with pieces of apple as a food source. To record their excavating behaviour using a Canon 6D Mark II camera 
with a Canon macro $100 \mathrm{~mm} f / 2.8$ lens (Canon Inc., Tokyo, Japan), a single female was placed on an arena with access to young acorns. As soon as the female started the interaction with an acorn, we started the video recording for one female.

\subsection{Scanning electron microscopy (SEM)}

We visualised the cuticular layers across cross sections of the rostrum using scanning electron microscopes Hitachi S-4800 at an accelerating voltage of $3 \mathrm{kV}$ and tabletop TM3000 SEM at an accelerating voltage of $15 \mathrm{kV}$ (Hitachi High-Tech Corp., Tokyo, Japan). For this purpose, two females and two males were fixed with $2.5 \%$ glutaraldehyde in $1 \times$ phosphate-buffered saline ( $\mathrm{pH}$ 7.4) (PBS) for one night. Then the samples were rinsed in PBS twice, dehydrated in ascending series of ethanol from $10 \%$ to absolute ethanol and dried using an E3100 critical point dryer (CPDA/Quorum Technologies Ltd., Kent, UK). We either transversely broke the rostra by bending them while holding with fine tweezers or cut the rostra using a razor blade into three regions (base, middle, apical), made them into small segments, and glued them perpendicularly to SEM stubs so that we could observe the cross sections. In addition, we prepared five air-dried females and six air-dried males in the same way to evaluate the variations among individuals. Then the samples were coated with gold-palladium (ca. $10 \mathrm{~nm}$ in thickness) using a Leica EM SCD500 high vacuum sputter coater (Leica Microscopy GmbH, Germany) before observation. Based on resulting micrographs, we measured thicknesses of epi\& exocuticle and endocuticle layers using the software FIJI [14]. Since the thicknesses are not constant at different locations within a cross section, we focused on the most upper and lower positions of each cross section, as representatives of the dorsal and ventral sides, respectively. Subsequently, we performed statistics using the software R 4.0.2 [15]. Considering that most of our datasets did not meet a criterion of normality, we compared measurements between females and males at various locations using the Wilcoxon rank-sum test.

\subsection{Material distribution analyses}

Following the method established by Michels and Gorb [16], we analysed material distributions of the rostrum using a ZEISS LSM 700 confocal laser scanning microscope (Carl Zeiss Microscopy GmbH, Jena, Germany). For this analysis, two females and one male were used to visualise material distributions along the rostrum. Additionally, we used two females and two males, to visualise material distributions within cross sections. First, living weevils were anaesthetised with carbon dioxide, and their rostra were removed in $1 \times$ PBS. The rostra were submerged in glycerine ( $\geq 99.5 \%$, free of water, Carl Roth GmbH \& Co. KG, Karlsruhe, Germany), placed on glass slides and covered with a coverslip. We visualised autofluorescence of the samples using four stable solid-state lasers with wavelengths of 405, 488, 555 and $639 \mathrm{~nm}$. Then for detection of selective emitted autofluorescence, we applied either a bandpass emission filter, transmitting light with wavelengths $420-480 \mathrm{~nm}$, or long pass emission filters, transmitting light with wavelengths $\geq 490, \geq 560$ or $\geq 640 \mathrm{~nm}$, respectively. Objective lenses with $2.5 \times$ [Objective LD LCI Plan-Apochromat, numerical aperture (NA) $=0.8], 2.5 \times($ EC EPIplan-NEOFLUAR, NA $=0.06$ ) and $20 \times($ Zeiss Plan-Apochromat, air immersion, $\mathrm{NA}=0.8$ ) magnification were applied. Following Michels and Gorb [16], we assigned blue, green, red (50\% saturation) and red (50\% saturation) to each micrograph and stacked them to create maximum intensity projections using the software Zeiss Efficient Navigation (ZEN) (Carl Zeiss MicroImaging $\mathrm{GmbH}$ ). According to Michels and Gorb [16], the resulting images can be interpreted as follows: (1) red coloured areas are well-sclerotised; (2) green coloured areas are less sclerotised; (3) blue coloured areas are less sclerotised and carry a relatively large amount of rubber-like protein, resilin. This interpretation has been validated for adhesive setae of a ladybug utilising nanoindentation under an atomic force microscope [17].

\subsection{Finite element simulation of the mechanical performance of the rostrum}

Finite element (FE) simulations were performed to investigate the effects of two key factors of the curvature and graded properties on the mechanical response of the weevil rostrum to the applied loads as follows. It is important to mention that our aim here is not to reproduce the exact mechanical behaviour of the rostra under loading, but rather to understand the role of the material and geometric features on their mechanical performance. Although our results enabled us to interpret the relative performance of the modelled rostra with confidence, the actual values of the obtained forces should be interpreted with caution.

\subsection{Analysis of the effect of rostrum curvature}

Similar to many other natural forms, such as horns, claws, tusks, etc., the weevil rostrum can be generated by moving a cross-sectional shape (likely a circle) along a path that is defined by a logarithmic spiral [18], described by the following equation

$r=r_{0} e^{-k \theta}$

where $r$ and $\theta$ are polar coordinates. $r_{0}$ and $k$ are the size and shape parameters, respectively. These two parameters and the range of the angle $\theta$ will be used to describe the length and curvature of the rostrum. $r_{0}=4.8 \mathrm{~mm}, k=0.7$ and $0 \leq$ 
a rostrum models with different curvatures
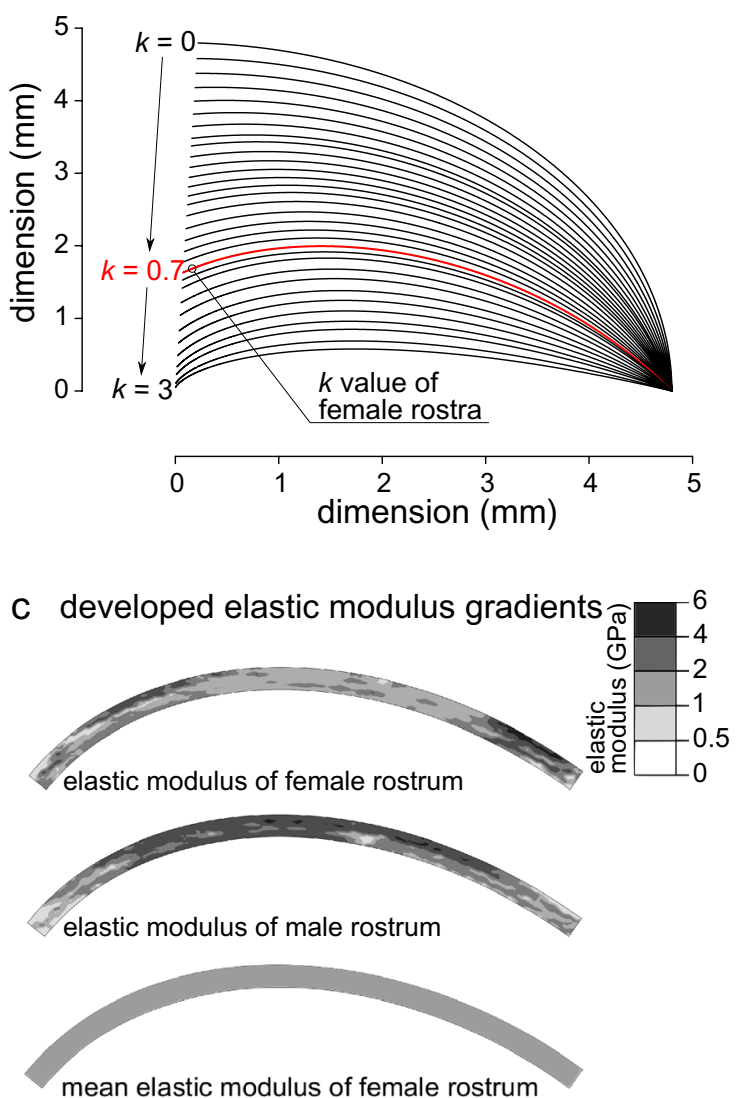

e simulated buckling \& bending

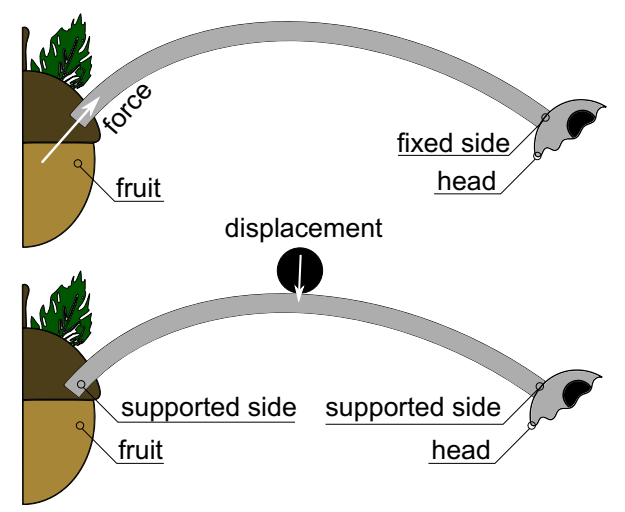

Fig. 2 Finite element simulation of the mechanical performance of the rostrum. a Models with different $k$ values, which represent rostra with different curvatures, were developed and analysed. The red curve represents the female rostra with a measured average $k$ value of $0.7(N=3)$. b Changes of the buckling force by changing the rostrum curvature. The model with a curvature similar to that of female rostra exhibits one of the highest buckling resistances. c Three elastic modulus gradients were assigned to the geometric model of the female rostrum. This included a gradient according to that observed

\section{b buckling force of curved models}
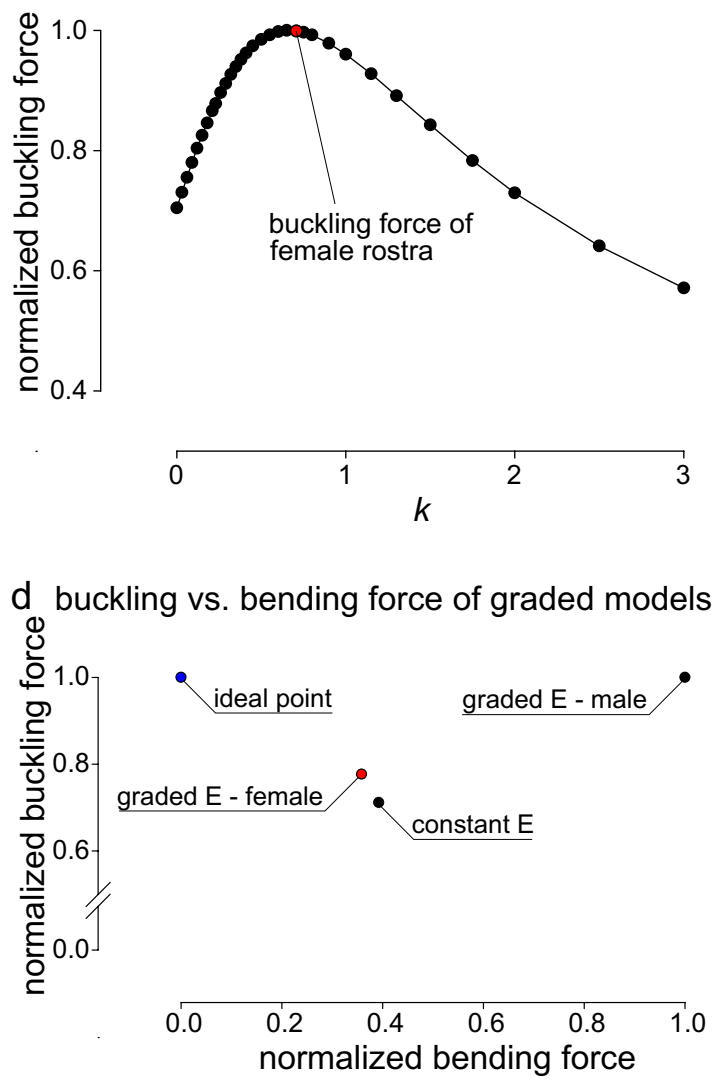

in the female rostrum, a gradient according to that observed in the male rostrum and a constant elastic modulus equal to the mean elastic modulus of the female rostrum. d Buckling force vs. bending force of the models with different elastic modulus gradients. $\mathrm{E}$ is the elastic modulus. e Loading and boundary conditions used in buckling (upper image) and in bending (lower image) simulations. In all panels, the base and the tip of the rostra are on the right and left sides, respectively. The force data were normalised with respect to the maximum values 
$\theta \leq 1.1 \mathrm{rad}$ are the parameters that can be used to reproduce the female rostrum (red line in Fig. 2a).

We changed the shape parameter, $k$, to develop a variety of rostrum models with different curvatures (Fig. 2a). The models were developed using the finite element software package ABAQUS/Standard v6.14 (Simulia, Providence, RI, USA). In this study, we used two-dimensional (2D) in-plane models, because although the rostra are three-dimensional (3D) structures, they are symmetrical in both sexes. We have also observed that, during the excavation process, the rostra experience only small lateral deformations. Therefore, we assumed that out-of-plane deformations of the rostra are negligible in comparison with their in-plane deformations. We expect that our 2D models can sufficiently reflect the influence of the geometry of the rostra on their mechanical performance. It is also important to mention that our aim here is not to estimate the exact deformations of the rostra under loading, but rather to test their performance in a comparative way. Hence, we expect that 2D models can provide reliable data for such a comparative analysis.

The models were meshed using the general-purpose four-node plane stress elements (CPS4R). We assigned the same elastic modulus of $1 \mathrm{GPa}$ to all the developed models. This is equal to the mean value of the elastic modulus of the female rostrum, which was estimated based on the CLSM image of the rostrum and using the nondestructive material characterisation method developed by Eshghi et al. [19]. For this purpose, we estimated the elastic modulus of 600 uniformly distributed points on the rostrum and then measured their mean value. The Poisson's ratio of the model was set as 0.3 , which is the same as that of many previously investigated biological materials [20] and has frequently been used for models of insect cuticle [21-23].

The models were used to simulate the buckling resistance of the rostra with a variety of curvatures during excavating egg chambers (Fig. 2e). To this end, the models were fixed at the base, and a unit load was applied to their tip. An eigenvalue buckling analysis was carried out to measure the critical load required to buckle the models [24].

\subsection{Analysis of the effect of graded properties}

To understand the role of the specific material gradient of the rostrum in its mechanical performance, we developed three FE models. The developed models had the same geometry as that of the female rostrum, but three different elastic modulus gradients: (i) an elastic modulus gradient as that of the female rostrum, (ii) an elastic modulus gradient as that of the male rostrum and (iii) a homogeneous elastic modulus equal to the mean elastic modulus of the female rostrum. The elastic modulus gradients of the female and male rostra were obtained based on their CLSM images and using the MATLAB-based program developed by Eshghi et al. [19]. The elastic modulus of the
Fig. 3 Scanning electron micrographs of mouthparts with the particular focus on the mandibles (red) in Curculio glandium. a-b Lateral view of the mouthparts. (c-n) Frontodorsal view of the mouthparts. c Left mandible and its posterior articulation; postcoila (the redcoloured region) is visible. $\mathbf{d}$ Right mandible and its postcoila (the circular region)
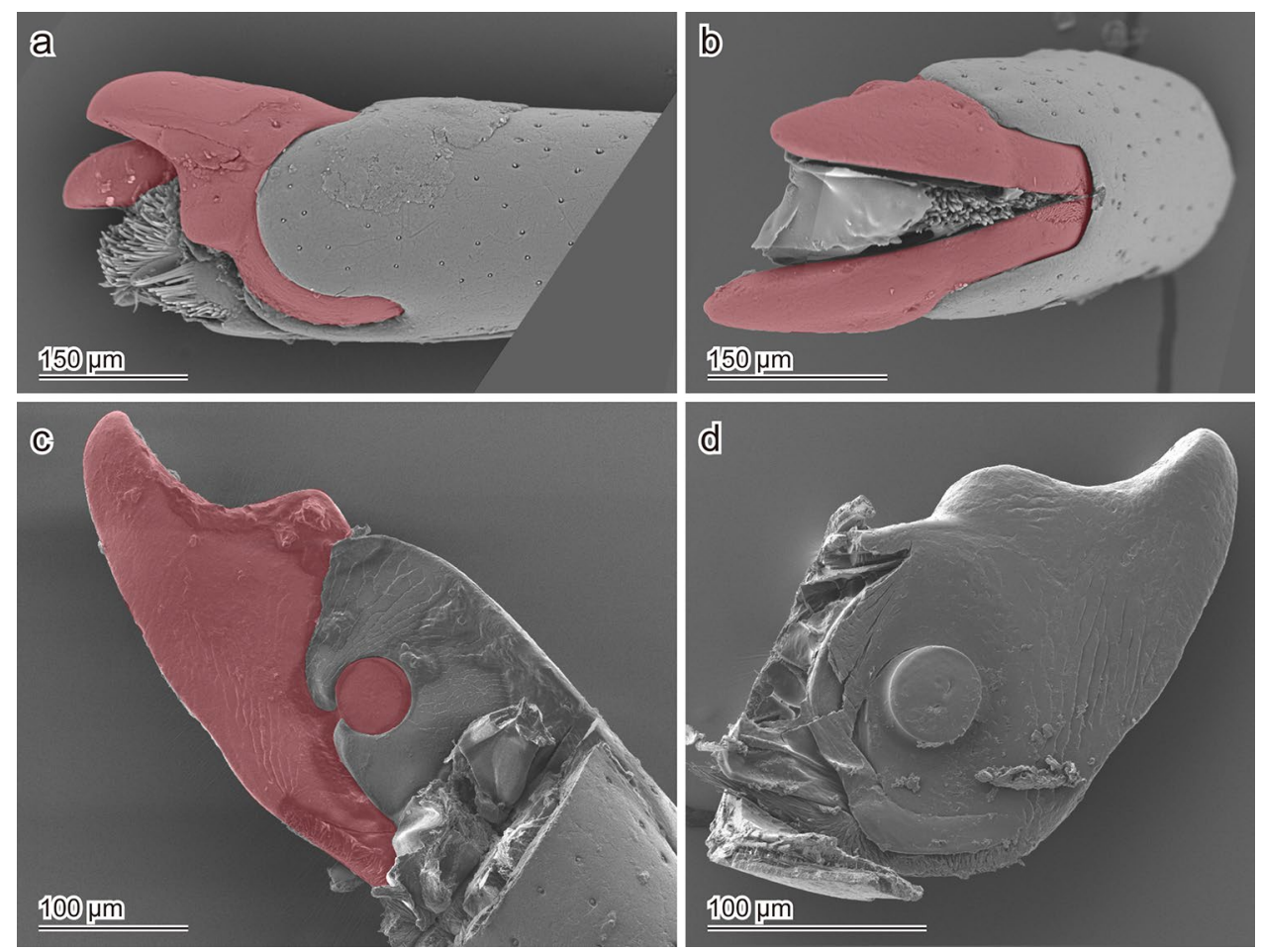

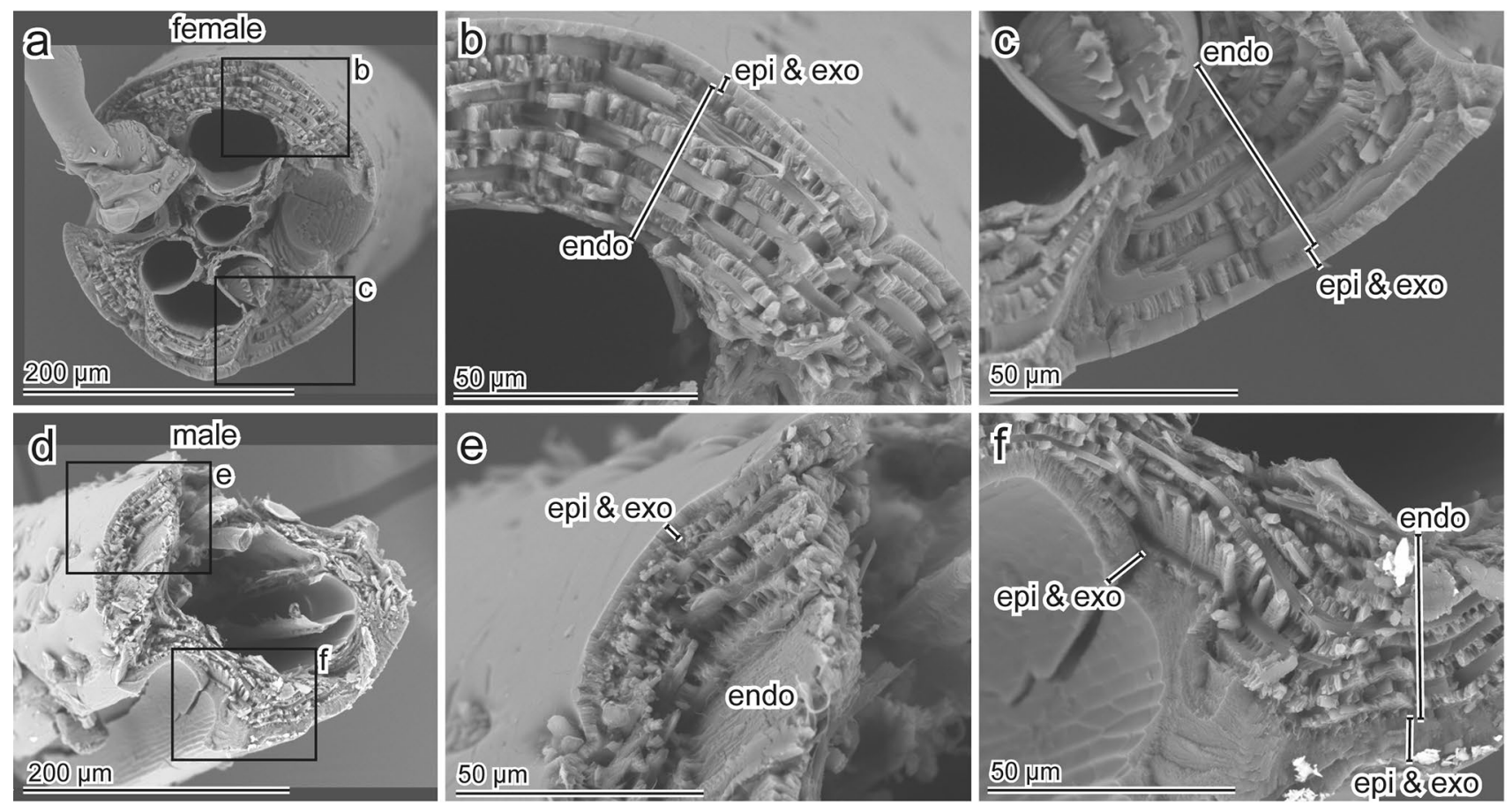

Fig. 4 Scanning electron micrographs of Curculio glandium rostra of the female a-c and male d-f. Cross sections at the base

female and male rostra ranged from $46 \mathrm{MPa}$ to $6 \mathrm{GPa}$ and from $25 \mathrm{MPa}$ to $5.6 \mathrm{GPa}$, respectively. The mean elastic modulus of the female rostrum was $1 \mathrm{GPa}$.

The developed models were subjected to two loading scenarios: buckling and bending. The simulation of the buckling was performed using the method described earlier. The simulation of the bending was carried out by fixing the vertical displacements at the two ends of the rostrum models and applying a displacement of $0.5 \mathrm{~mm}$ to their middle point (Fig. 2e). The displacement was applied using a cylindrical object situated on the uppermost side of the rostrum. A 'hard contact' and a 'frictionless contact' were used to define the normal and tangential behaviour between the object and the rostrum, respectively. The defined hard contact prevents the penetration of the contact pairs into each other.

\section{Results}

\subsection{Rostrum morphology and excavating behaviour}

The female rostrum in $C$. glandium is approximately twice as long as the male one and tends to be steeply curved ventrally [females: $6.01 \pm 0.52$ (mean \pm s.d., $N=3$ ), males: $2.94 \pm 0.27$ $(\mathrm{N}=3)$ ] (Fig. 1a). The mouthparts are situated at the tip of the rostrum, and the mandibles lie parallel to each other
(Fig. 3, Appendix video 1) as known in derived weevil lineages [4]. One of the two mandibular articulations to the rostrum body, postcoila, was observed (Fig. 3). From the morphological point of view, it is obvious that the mandibles move only dorsoventrally.

We recorded the excavating behaviour of a female (Appendix video 1, Fig. 1b-e). During the excavation process, the mandibles are not visible, but apparently, females can move the mandibles quite fast dorsally and ventrally (Appendix video 1 at 00:15). The female often rotated her entire head at the neck region from side to side, and once she turned almost $180^{\circ}$ pivoting on her rostrum. Sometimes the rostrum was ventrally and dorsally bent during the excavating behaviour (Appendix video 1 at 01:18-01:25). In comparison with this dorsoventral rostrum deformation, a lateral deformation was not striking at all. In the end, the weevil excavated a long channel in the acorn, so that the female could penetrate her entire rostrum in the excavated lumen. Then the female pulled out her rostrum by fully stretching the legs, turned and started inserting the ovipositor to lay an egg in each channel. The whole excavation process with intact acorns usually lasts ca. $1 \mathrm{~h}$ or longer (M.R. pers. observ.).

\subsection{Ultrastructure of the rostrum}

The rostrum is almost round in the cross section, except for the antennal socket region, at which it is dumbbell-shaped 


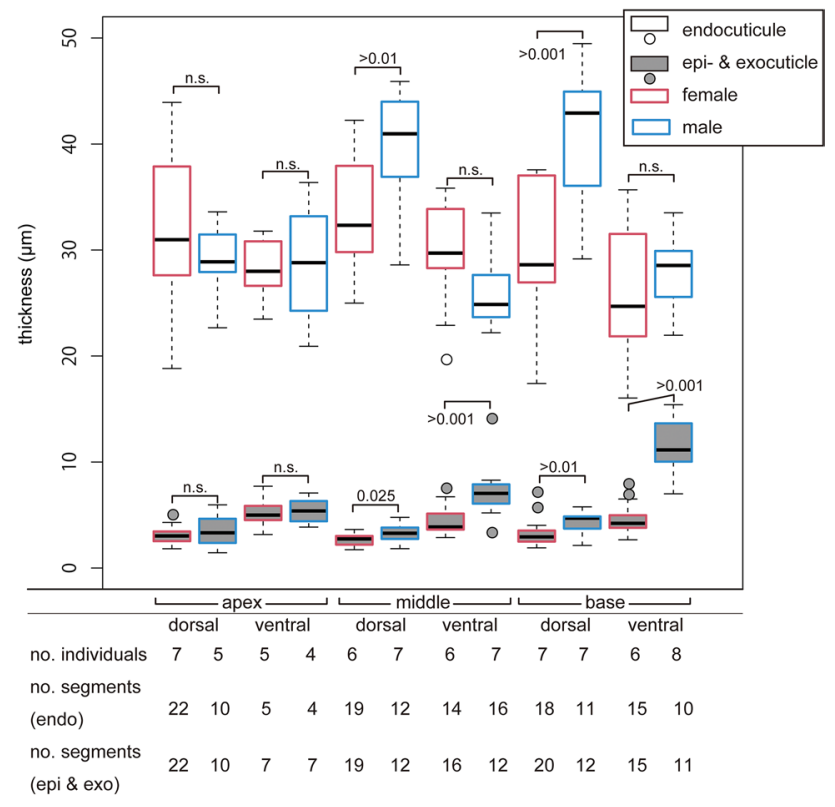

Fig. 5 Measurements of the thicknesses of the epi- \& exocuticle and endocuticle of Curculio glandium. The number of observed individuals and fractured segments was listed at the bottom

(Figs. 4 and 6). The diameter of the rostrum is overall homogeneous, but the basal part is slightly wider in both sexes. The cuticle fibres, so-called macrofibres [25], of the endocuticle, are visible in fractured surfaces (Fig. 4). The fibres form lamellae, and each of them seems to be oriented $\pm 45^{\circ}$ with respect to the longitudinal axis of the rostrum.

Considering that we examined weevil samples that were prepared using two different methods (fixed with glutaraldehyde vs. air-dried) to measure cuticular layers, we first compared our measurements between the treatments (Appendix Figs. 1 and 2). Due to the small sample size, we did not perform statistics, but we did not see any striking difference in the results of the two measurements. Therefore, we pooled all data (Fig. 5). The thickness of the epi$\&$ exocuticle and endocuticle varies among different areas of the rostrum and between the sexes (Fig. 5). The exocuticle of the male is thicker in the middle and basal regions comparing with the apical region (Wilcoxon rank sum test: $W_{\text {middle,dorsal }}=59, N_{\text {female }}=19, N_{\text {male }}=12, P=0.025$; $W_{\text {middle, ventral }}=25, N_{\text {female }}=16, N_{\text {male }}=12, P<0.001$; $W_{\text {base } \text {, dorsal }}=51, N_{\text {female }}=20, N_{\text {male }}=12, P<0.01$; $\left.W_{\text {middle,ventral }}=1, N_{\text {female }}=15, N_{\text {male }}=11, P<0.001\right)$. The male endocuticle thickness was thicker only at the dorsal side of the middle and basal regions of the rostrum (Wilcoxon rank sum test: $W_{\text {middle,dorsal }}=44, N_{\text {female }}=19$, $N_{\text {male }}=12, P<0.01 ; W_{\text {base } \text { dorsal }}=21, N_{\text {female }}=18$, $\left.N_{\text {male }}=11, P<0.001\right)$.

\subsection{Graded material of the rostrum}

Material gradients were detected along the longitudinal axis of the rostra in both sexes (Fig. 6a, e). The outer surface of the male rostrum was dominated by the red coloured autofluorescence in general and, therefore, is likely to be wellsclerotised (Fig. 6a). The female rostrum, in contrast, was dominated by the green coloured autofluorescence, particularly in its middle part (Fig. 6e). Hence, we expect the middle part of the rostrum to be less-sclerotised than the apex and base, which seem to be well-sclerotised.

Gradients in the sclerotisation level were also observed within the cross sections of the rostra (Fig. $6 b-d, f-j$ ). In the male, the thin outer epicuticle layer appeared in yellowishgreen, the exocuticle was dark red, and the endocuticle was whitish-green (Fig. 6b-d). These observations suggest that the exocuticle is likely to be well-sclerotised, and the epicuticle and endocuticle are comparatively less-sclerotised. In the female, both the epicuticle and exocuticle were visualised with yellowish-green (Fig. 6f-i), except at the base, in which the exocuticle appeared in dark red (Fig. 6j). In contrast to the male rostrum, the endocuticle of the female rostrum, except at the base, was red (Fig. 6f-i). It appears that the endocuticle of the female rostrum is even more sclerotised than the exocuticle. The inner part of the endocuticle layer, particularly on the ventral side, showed the blue coloured signal, which suggests the dominance of resilin there (Fig. 6f-i). Only at the basal part, the endocuticle is less sclerotised than the epicuticle and exocuticle of the same region (Fig. 6j).

\subsection{Behaviour of the rostrum in buckling and bending}

Buckling of the rostrum models with various curvatures was simulated numerically. Figure $2 \mathrm{~b}$ compares the critical force needed to buckle (i.e. the buckling force) the developed models. The female rostrum has an average $k=0.7(N=3$, the red data point in Fig. 2b). As seen here, although an increase of the shape parameter, $k$, led to an increase of the buckling resistance of the rostrum models for $0 \leq k \leq 0.65$, the buckling resistance continuously decreased with $k>$ 0.65 . The maximum buckling force was achieved in a rostrum with $k=0.65$. Surprisingly, this is very close to the curvature of the real female rostrum $(k=0.7)$.

We also investigated the effect of the specific graded properties of the rostrum on their mechanical behaviour, by applying three different elastic modulus gradients to the geometric model of the female rostrum. The used elastic modulus gradients included (i) a gradient according to that observed in the female rostrum, (ii) a gradient according to that of the male rostrum and (iii) a homogeneous 
Fig. 6 Confocal laser scanning micrographs of Curculio glandium rostra of the female a-d and the male $\mathbf{e}-\mathbf{j}$. The blue-coloured structures situated in the lumen of the rostra are tendons. We excluded the tendons from adjustments of signal intensities for the CLSM analyses, and therefore the colour of these tendons is oversaturated

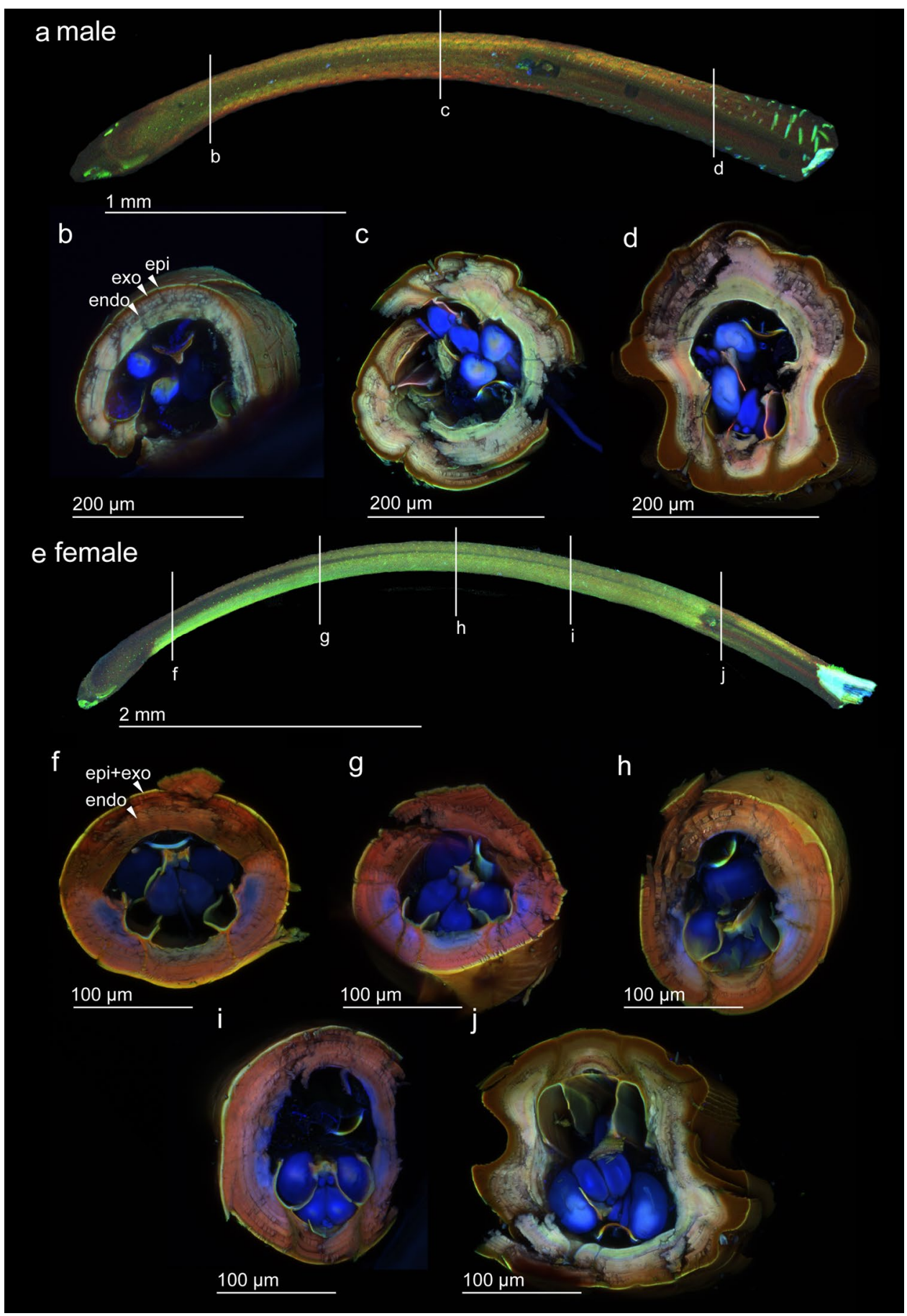

elastic modulus equal to the mean modulus of the female rostrum (Fig. 2c). The results obtained from the simulation of the buckling and bending of the models showed that the model with a graded elastic modulus as that of the male rostrum had the highest buckling force among others (Fig. 2d). This is the force required to initiate rostrum buckling. The buckling force of the model with the female's elastic modulus gradient was lower than that of the model with the elastic modulus gradient of the male rostrum (0.8 times), but higher than that of the model with a constant elastic modulus (1.1 times). The force required to bend the models for the same displacement was also the highest for the model with a graded elastic modulus as that of the male rostrum (Fig. 2d). Here the model with a graded elastic modulus as that of the female rostrum had the lowest bending force than the others. 


\section{Discussion}

The structure and configuration of beetle mouthparts are generally conservative, despite a broad spectrum of food sources they feed on [26]. Weevils are no exception although they have developed rostra [4]. However, the mandible arrangement is shifted in derived lineages and they lay vertically as seen in $C$. glandium. Moreover, in Curculio species, the mandibular adductor tendons are reduced and the abductor tendons are enlarged [4]. These modifications may be associated with the fast movement of the mandibles observed in the present study. Although the mandible movements can influence the excavation by rasping or scratching substrates, the entire rostrum is likely to play the major role. We have observed notable dorsoventral deformations of the rostra while females rotate their heads. Our observations suggest that females actively push their rostra into acorns and seemingly control the orientation of excavating channels. In addition to this, females of the brentid weevil species Rhopalapion longirostre excavate channels while carrying male partners, and it can happen that another male tries to copulate at the same time [27]. The females, therefore, may encounter unexpected loads and be abruptly moved during excavation. Our results suggested that the elongated female rostrum is highly adapted to its function as an excavation tool. The present study, for the first time, shows that the sexual dimorphism of acorn weevils is not only reflected by the different lengths of their rostra, but also by striking differences in the rostra morphology and sclerotisation level.

One of the remarkable adaptations was found in the rostrum curvature. In comparison with the male rostrum, the female rostrum tends to be more ventrally curved. We showed that the curvature plays a strong role in the buckling resistance of the rostrum. Interestingly, we found that the curvature of the female rostrum has been adapted to provide it with a maximal buckling resistance. Considering that the curvature of the female rostrum is not constant among species, and even within the genus Curculio [6], future research should examine the relationship between the curvature and buckling resistance of the female rostra in relation to rostrum lengths.

We also found that the female and male rostra show sex-specific material gradients both along their lengths and across the cross sections (Fig. 6). We included these material gradients in our FE models and found that the material gradient of the female rostrum is adapted to provide it with a high buckling resistance while maintaining a low bending resistance (Fig. 2d). This is an interesting finding, which helps us to understand how the rostrum works in practice. To excavate an egg channel with no interruption, the buckling failure in the female rostrum should be prevented. At the same time, the curved rostrum should be straightened to reduce the tangential force component and, thereby, to increase the normal component of the applied force. Moreover, to make the channel as deep as possible, the rostrum should be more or less straightened (as seen in Appendix video 1). This observation means that while the buckling resistance needs to be maximised, the bending resistance should be minimised, so that the rostrum can function as an excavation tool. However, these two properties are mutually inclusive; the increase in one of them increases the other one. Our results suggest that a combination of a specific graded elastic modulus and a near 'optimal' curvature enables the rostra of the female $C$. glandium to reach a balance between a maximal buckling resistance and minimal bending resistance.

Jansen et al. [9] hypothesised that the specific layered structure of the endocuticle, i.e. seemingly pseudo-orthogonally oriented macrofibre laminae, prevents the propagation of surface cracks and, thereby, contributes to the rostrum toughness. Our observations of the rough fracture surface, delamination and fibre pull-outs in the endocuticle of the fractured samples support this hypothesis (Fig. 4). Moreover, Jansen et al. [9] documented that the exocuticle-rich occipital sulci situated at the ventral side are the most likely part, where critical failure initiates. Our findings suggest that the resilin-rich patches, which were observed only on the ventral side of the endocuticle of the female rostrum (Fig. 6), contribute to diminish critical stresses occurring at the exocuticle-rich occipital sulci in this region. This remains hitherto to be a hypothesis and has to be further critically tested.

In addition to the generally thicker cuticle of the rostrum in males than in females (Fig. 5), another noticeable sexual dimorphism is the plausibly well-sclerotised endocuticle of the female rostrum. This is evident by our CLSM observations of a noticeably stronger red autofluorescence in the endocuticle of the female rostrum in comparison with that of the male (Fig. 6). Although it has to be proven by measuring elastic modules in $C$. glandium, this could additionally be confirmed by the results of a previous study on the mechanical properties of the rostrum of the weevil C. longinasus [12]. The nanoindentation measurements on samples embedded in resin indicated a relatively high elastic modulus of the rostrum endocuticle, which lies within the range of that of the well-sclerotised cuticle. The occurrence of a highly sclerotised endocuticle is very rare in insects and has been reported only once before except for the rostrum. Wang et al. $[28,29]$ showed that the endocuticle of the elytra of the curculionoid weevil species, Pachyrhynchus sarcitis, is also strongly sclerotised. The presence of a sclerotised endocuticle is likely to be a strategy to enhance the mechanical strength of the rostrum, i.e. its load-bearing capacity. In weevils, a specific combination of cuticular structures 
is known [25]. Such a particular condition of the cuticle in weevils can have promoted the development of the unique rostrum in weevils, which requires mechanical stability. To test the hypothesis, we need to examine the ultrastructure of the rostrum in Phytophaga (weevils, leaf beetle, and longhorn beetles) comparatively, including the New Zealand giraffe weevil, Lasiorhynchus barbicornis, where males have a much longer rostrum than females [30].

Future studies should focus on quantitative comparisons of the material properties of the rostra with respect to those of acorns, on which weevils feed. For instance, previous studies have found a correlation between the mechanical properties of dragonfly ovipositors and their host plants, where female dragonflies lay eggs [31]. A similar trend may be present between the mechanical properties, in particular the elastic modulus, of the weevil rostra and those of the plant substrates.

\section{Conclusions}

The functionality of the rostrum, as a mechanical tool for making channels into fruits, is the result of a compromise between the stiffness and flexibility. This is due to two key reasons. On the one hand, to penetrate into fruits and make a deep egg-laying channel, the originally curved rostrum has to be flexible enough to straighten reversibly. On the other hand, to avoid an interruption in the excavation process, the rostrum should be stiff enough to prevent buckling. Our results suggest that the compromise between the stiffness and flexibly in the rostrum of the female weevil $C$. glandium is obtained by two key material and geometric features: a specific graded elastic modulus and a near 'optimal' curvature.

\section{Supporting materials}

All supplementary materials, including the Appendix Figure 1, Appendix Figure 2 and Appendix Video 1, are accessible via the following link:https://figshare.com/s/de0d0 ad24ed525c32661

Acknowledgements We thank Esther Appel and Dr. Alexander Kovalev (Kiel University, Germany) for sputter-coating the cuticle samples.

Author contributions H.R. and Y.M. contributed to conceptualisation through discussion with all co-authors; Y.M., M.J., and H.R contributed to methodology through discussion with all co-authors; Y.M., M.J., M.R., B.S.M., and H.R. were involved in investigation; M.R., A.D., and S.N.G. provided the resources; Y.M. and H.R. contributed to data curation; Y.M., M.J., M.R., S.N.G., and H.R contributed to data interpretation; Y.M. and H.R. contributed to writing-original draft preparation; , Y.M., M.J., M.R., B.S.M., and S.N.G. contributed to writing-review and editing; Y.M. and H.R were involved in visualisation; A.D., S.N.G., and H.R were involved in supervision; Y.M. and H.R. contributed to project administration; Y.M. and M.J contributed to funding acquisition. All authors have read and agreed to publish the manuscript.

Funding Open Access funding enabled and organized by Projekt DEAL. This research was funded by the German Research Foundation (DFG grant no. MA 7400/1-1) to YM and by Federal State Funding at Kiel University to MJ.

\section{Compliance with ethical standards}

Conflicts of interest The authors declare no conflict of interest.

Availability of data and material Measurements are available as supplementary materials. Other data are available from the corresponding author upon reasonable request.

Code availability Not applicable.

Open Access This article is licensed under a Creative Commons Attribution 4.0 International License, which permits use, sharing, adaptation, distribution and reproduction in any medium or format, as long as you give appropriate credit to the original author(s) and the source, provide a link to the Creative Commons licence, and indicate if changes were made. The images or other third party material in this article are included in the article's Creative Commons licence, unless indicated otherwise in a credit line to the material. If material is not included in the article's Creative Commons licence and your intended use is not permitted by statutory regulation or exceeds the permitted use, you will need to obtain permission directly from the copyright holder. To view a copy of this licence, visit http://creativecommons.org/licenses/by/4.0/.

\section{References}

1. R.S. Anderson, Mem. Entomol. Soc. Wash. 14, e114 (1995)

2. R.G. Oberprieler, A.E. Marvaldi, R.S. Anderson, Zootaxa 1668, 491 (2007)

3. A.E. Marvaldi, A.S. Sequeira, C.W. O'Brien, B.D. Farrell, Syst. Biol. 51, 761 (2002)

4. S.R. Davis, BioRxiv, 287185 (2018)

5. S.R. Davis, Bull. Am. Mus. Nat. Hist. 2017, 1 (2017)

6. J. Hughes, A.P. Vogler, Evolution 58, 1971 (2004)

7. H. Toju, T. Sota, Biol. Lett. 2, 539 (2006)

8. H. Toju, T. Sota, Am. Nat. 167, 105 (2006)

9. M.A. Jansen, J. Williams, N. Chawla, N.M. Franz, Adv. Mater. 31, e1903526 (2019)

10. A. Sakes, D. Dodou, P. Breedveld, Bioinspir. Biomim. 11, 21001 (2016)

11. U. Cerkvenik, D. Dodou, J.L. van Leeuwen, S.W.S. Gussekloo, Biol. Rev. Camb. Philos. Soc. 94, 555 (2019)

12. S.S. Singh, M.A. Jansen, N.M. Franz, N. Chawla, Mater. Charact. 118, 206 (2016)

13. M.A. Jansen, S.S. Singh, N. Chawla, N.M. Franz, J. Struct. Biol. 195, $139(2016)$

14. J. Schindelin, I. Arganda-Carreras, E. Frise, V. Kaynig, M. Longair, T. Pietzsch, S. Preibisch, C. Rueden, S. Saalfeld, B. Schmid, J.Y. Tinevez, D.J. White, V. Hartenstein, K. Eliceiri, P. Tomancak, A. Cardona, Nat. Methods 9, 676 (2012)

15. R Core Team (2020) R: A language and environment for statistical computing. R Foundation for Statistical Computing, Vienna, Austria. URL https://www.R-project.org/. 
16. J. Michels, S.N. Gorb, J. Microsc. 245, 1 (2012)

17. H. Peisker, J. Michels, S.N. Gorb, Nat. Commun. 4, 1661 (2013)

18. D.W. Thompson, On Growth and Form (Cambridge University Press, Cambridge, 1942), p. 116

19. S.H. Eshghi, M. Jafarpour, A. Darvizeh, S.N. Gorb, H. Rajabi, J.R. Soc, Interface 15, 20180312 (2018)

20. S.A. Wainwright, W.D. Biggs, J.M. Gosline, J.D. Currey, Mechanical Design in Organisms ( $p$ 436) (Princeton University Press, Princeton, 1982).

21. H. Rajabi, M. Jafarpour, A. Darvizeh, J.-H. Dirks, S.N. Gorb, J.R. Soc, Interface 14, 20170310 (2017)

22. A. Toofani, S.H. Eraghi, M. Khorsandi, A. Khaheshi, A. Darvizeh, S. Gorb, H. Rajabi, Acta Biomater. 110, 188 (2020)

23. M. Jafarpour, S. Eshghi, A. Darvizeh, S. Gorb, H. Rajabi, J.R. Soc, Interface 17, 20200378 (2020)

24. ABAQUS, ABAQUS v6.7. Analysis user's manual. Providence, RI: Simulia; 2007.
25. T. van de Kamp, A. Riedel, H. Greven, Arthropod Struct. Dev. 45, 14 (2016)

26. R.G. Beutel, M. Yavorskaya, in Insect Mouthparts, ed. H.W. Krenn, (Springer, New York, 1973), pp. 387-418

27. G. Wilhelm, S. Handshuh, J. Plant, H.L. Nemeschkal, Biol. J. Linn. Soc. 104, 642 (2011)

28. L.-Y. Wang, H. Rajabi, N. Ghoroubi, C.-P. Lin, S.N. Gorb, Front. Physiol. 9, 1410 (2018)

29. L.-Y. Wang, M. Jafarpour, C.-P. Lin, E. Appel, S.N. Gorb, H. Rajabi, Soft Matter 15, 8272 (2019)

30. C.J. Painting, G.I. Holwell, PLoS ONE 8, e82467 (2013)

31. N. Matushkina, S.N. Gorb, Zoology 110, 167 (2007)

Publisher's Note Springer Nature remains neutral with regard to jurisdictional claims in published maps and institutional affiliations. 